\title{
Mitigating Psychological Impact: The Experience of Korean Disaster Management
}

\author{
Kyoo-Man $\mathrm{Ha}^{1}$ (1)
}

Received: 30 January 2020 / Accepted: 24 June 2020 / Published online: 2 July 2020

(c) Springer Science+Business Media, LLC, part of Springer Nature 2020

\begin{abstract}
The psychological impact of disasters has not been adequately addressed in Korea. This research aims to evaluate how Korea should improve its management of the psychological impact of disasters toward the ultimate goal of effective disaster management. Qualitative content analysis is the main method applied. Nonprofessional management is compared with professional management by considering governments, psychology specialists, disaster victims, and local communities. The main finding is that Korea must change its current management style from nonprofessional to professional. Neighboring nations need to implement supplementary measures toward adopting a systematic approach that considers all phases of the disaster management cycle, emergency planning, and a long-term approach. The value of this study lies in its comprehensive examination of the issue of psychological impact from the perspective of disaster management in Korea.
\end{abstract}

Keywords Physical impact $\cdot$ Social impact $\cdot$ Psychology specialists $\cdot$ Disaster victims $\cdot$ Social support

\section{Introduction}

People experience the psychological impact of a disaster before, during, and after the actual occurrence of the disaster event. In particular, during the period of initial disaster response, the behavior and mentality of the public are temporarily disrupted due to stress. In the following period, not only fear but also physical deprivation is experienced (McGonagle 1964). In the end, people have to overcome diverse emotional disturbances. In this regard, many countries have started to pay attention to how other nations manage the psychological impact of disasters.

South Korea (hereinafter Korea) is no exception when considering such psychological impact, given that Koreans have been repeatedly affected by disasters, including the current outbreak of coronavirus disease 2019 (COVID-19). Major stakeholders have thus far generally focused on managing the physical impact of disasters, such as decreasing the loss of human lives and the amount of economic damage. In fact, only about $37 \%$ of Koreans substantially recognize

Kyoo-Man Ha

ha1999@hotmail.com

1 Department of Public Policy and Management, Pusan National University, 2, Busandaehak-ro 63beon-gil, Geumjeong-gu, Busan 46241, Korea the issue of psychological impact in the context of disaster management, as shown in Fig. 1. Given this scenario, the present research addresses the question of how Korea deals with the psychological impact of disasters.

This study aims to determine how the management of the psychological impact of disasters in Korea can be improved and to draw lessons for neighboring nations toward the ultimate goal of mitigating such impact. Nonprofessional and professional styles of management are compared considering four variables: government policy, psychology specialists' efforts, disaster victims' needs, and local community support.

\section{Literature Review}

Nonprofessional and professional management are regarded as opposite concepts in this research. Nonprofessional management, which is repetitive or manual in nature, deals with the psychological impact of disasters as a temporary career or even in inappropriate ways and is therefore amateurish. In contrast, professional management, which is characterized by specialized knowledge or advanced skills, deals with psychological impact as a permanent career (Stopper 2017). Thus, professional management addresses psychological impact according to a code of conduct or ethics. 


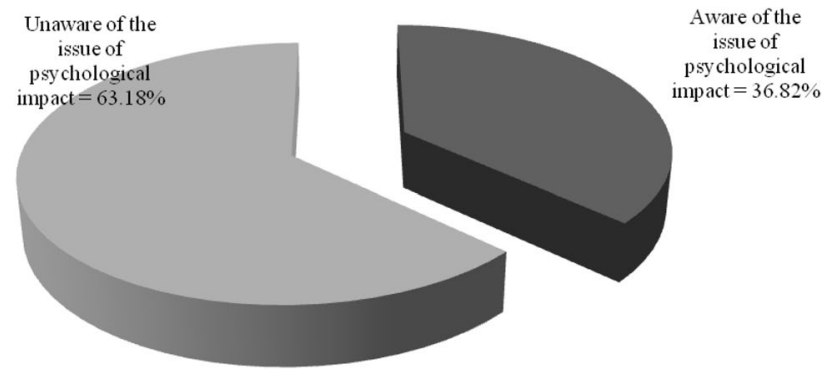

Fig. 1 Korean awareness of the psychological impact of disasters. Source: (Ansan Mental Health Trauma Center 2017)

In the twenty-first century, several nations have often addressed psychological impact in relation to disaster management. However, the effectiveness of psychological intervention has not been widely recognized (Gray et al. 2004). Notwithstanding, the psychological impact of a disaster has several symptoms, such as memory lapses, depression, substance use disorders, anxiety, stress, violence, interpersonal dysfunction, decreased productivity, isolation, post-trauma syndrome, and other emotional or psychological traumas. Without appropriate treatment, these symptoms may become aggravated and, in extreme cases, may lead some individuals to commit suicide (Tachibana et al. 2014; Lebowiz 2017). The rate of suicide is temporarily increased immediately after either a natural disaster or a manmade emergency. In addition, changes in interpersonal relationships or economic burdens may cause an increase in the suicide rate. Hence, constant monitoring of other nonfatal psychiatric conditions, as well as suicide condition, is necessary to decrease the suicide rate.

Some major issues regarding psychological impact have been traditionally discussed in the field of disaster management (Lindell and Prater 2003). For instance, decreasing the psychological impact, as well as the human loss and economic damage, is usually mentioned as part of the goals of disaster management. In addition, the field of disaster management frequently considers two kinds of impact resulting from disasters, that is, physical and social. Physical impact includes human loss and economic damage, whereas social impact includes psychological as well as sociopolitical, socioeconomic, and sociodemographic impact.

Fundamentally, an individual requires at least three of several components to deal with the psychological impact of a disaster: mental toughness, nutrition, and local culture. First, an individual needs mental toughness or competence, which may develop from previous experiences of coping with psychological impact (West and Albrecht 2007; Swann et al. 2016). When an individual has a strong motivation, personality, or cognitive mechanism, he or she may achieve a positive psychological outcome earlier, more flexibly, or more efficiently compared with a person without these attributes.

Second, an individual needs to have the appropriate amount of nutrient supplements to deal with the psychological impact of a disaster (Kaplan et al. 2015). Nutrition may not seem to be directly related to psychological impact given that the former is easily observable and the latter is not. However, without the necessary mineral and vitamin supplements to ensure good health, no individual would be able to recover quickly from the psychological impact of a disaster. Similarly, not only a healthy diet but also adequate sleep is essential to deal with such impact.

Third, from a broad perspective, local culture plays a role in enabling an individual to deal flexibly with the psychological impact of a disaster (Jogia et al. 2014; Belzen 2019). Local culture includes regional traditions, values, local history, and other factors that may affect the extent of one's vulnerability to psychological impact. Thus, cultural competence is highlighted as capable of positively influencing the psychological response or recovery of an individual in a community. In particular, social cohesion, which is the willingness of local residents to cooperate with one another for their survival, plays a robust role in communities not only in preparing for but also in responding to various disasters. Thus, the cultural variation among many regions or even within a given location can affect the extent of psychological impact.

Compared with physical health impact, the psychological impact of disasters may last longer or be more severe. However, many studies on the subject in the international community were done over a short period, particularly within the first 2 years after the occurrence of a specific disaster (Tanaka et al. 2016). Mainly because the psychological impact of a disaster is not easily noticeable or measurable, many researchers may not follow up on the related effects over the long term. Furthermore, previous studies on psychological impact have encountered certain limitations (Whittle et al. 2012), such as the difficulty of accurately identifying those individuals who have been affected by disasters.

Given the above situation, some researchers have provided important insights for studies on the psychological impact of disasters (Nomura et al. 2016). One such insight is that, in addressing such impact on individuals, the field of disaster management needs to apply intervention as early as possible and over the long term. According to $\mathrm{Hu}$ et al. (2010), about $75 \%$ to $80 \%$ of disaster victims who present acute stress disorder develop post-traumatic stress disorder, thus the need to provide early psychological intervention. In doing so, psychologists or medical staffs must obtain the trust of disaster victims by addressing coping skills. Simultaneously, psychological intervention should be systematically carried out over several years. Psychologists may provide long-term social support for disaster victims, 
such as making a close relation between disaster victims' psychological relief and social aggregation. Without such intervention, many individuals would suffer a higher degree of mental disorder.

The field of disaster management needs to apply various strategies in studying psychological impact, in particular toward eliminating the difficulties or limitations regarding this issue (Cox et al. 2017). For example, the field must not only explore the subject of psychology but also apply knowledge from multiple disciplines. Because the human psychology is so complicated, the field must efficiently apply diverse theoretical disciplines, including medicine, nursing, organization, and management.

In Korea, some accredited researchers have started to carry out studies on the psychological impact of disasters (Chae et al. 2005; Choi and Oh 2014). When the ferry MV Sewol sank in 2014, many students from Danwon High School were drowned to death. A research team that attempted to empirically compare the psychological impact between the Danwon area and a remote community found that medical doctors prescribed antidepressants at a $5.6 \%$ higher rate to residents in the Danwon area compared with those in the remote community (Han et al. 2017).

Nevertheless, there is not enough research on psychological impact or its management in the Korean field of disaster management. The majority of accredited researches have been in the areas of trauma psychology, psychiatry, and mental health. Therefore, the present work has particular value in that it approaches the issue of psychological impact in relation to disaster management. By considering aspects of disaster management, as well as psychology, psychiatry, and related fields, this research attempts to investigate the Korean experience as a pioneering work.

\section{Methodology}

Quantitative content analysis has been popularly used in the study of psychology, the subject being considered a science, through psychological experiments, correlational techniques, and other quantitative data studies. Nevertheless, qualitative content analysis has increased in importance in the field of psychology in the past decades (Gelo et al. 2008) due to some weaknesses of quantitative content analysis, such as defective measurement, variable ambiguity, and lack of meaningful interpretation.

Disaster management is a challenging subject to comprehend because it requires the fair inclusion of various aspects of personal, organizational, systematic, national, and international perspectives. Without the use of effective methodological tools, such as qualitative content analysis, the topic of disaster management could not address the targeted theme (Othman and Beydoun 2013).
The present work applied qualitative content analysis as the main methodology. Reports were identified, and the appropriate qualitative texts were interpreted to support the key tenet of the research. Internationally known search engines, such as ScienceDirect, EBSCOhost (ASC), Oxford University Press, and Google Scholar, were used to find appropriate text data. Some Korean search engines, including KISS, DBpia, and government websites, were also used.

The process of qualitative content analysis consisted of four steps (Hall 2017). The first step was the careful reading of the text materials. The second step was the definition of the units of analysis or categories to include personal motivation, organizational reaction, and disaster management frames. These categories were drawn from various behaviors and customs and from the existing literature. The third step was the coding of text materials by marking or highlighting. The fourth step was the interpretation and rewriting of the findings. The present work did not apply linear data extraction.

The research design defined two styles of managing the psychological impact of disasters in Korea, namely, nonprofessional and professional management. As shown in Fig. 2, these two styles were compared in terms of psychological impact mitigation. The management style better suited to Korea was then determined, after which important lessons for neighboring nations, as well as for Korea, were drawn.

The issue of psychological impact in the field of disaster management is influenced by diverse variables depending on the individual perspective (Smith et al. 2014). Based on the extensive literature review, four comparative variables in Korea were selected: government policy, psychology specialists' efforts, disaster victims' needs, and local community support. There is sufficient reason to consider each of these factors as a comparative variable. Governments are major stakeholders in the field of disaster management, whereas psychology specialists, as partners of the government, provide direct treatment to psychologically affected individuals. Disaster victims are the main parties affected by psychological impact and its accompanying symptoms. The local community as a close partner of the government includes various members, such as religious groups and residents.

\section{Nonprofessional Management of Psychological Impact}

\section{Government Policy}

The Ministry of the Interior and Safety (MOIS; previously the Ministry of Government Affairs and Security) at the central government level did not recognize the issue of psychological impact until the sinking of the ferry MV Sewol in 2014. Although the MOIS has provided policy 


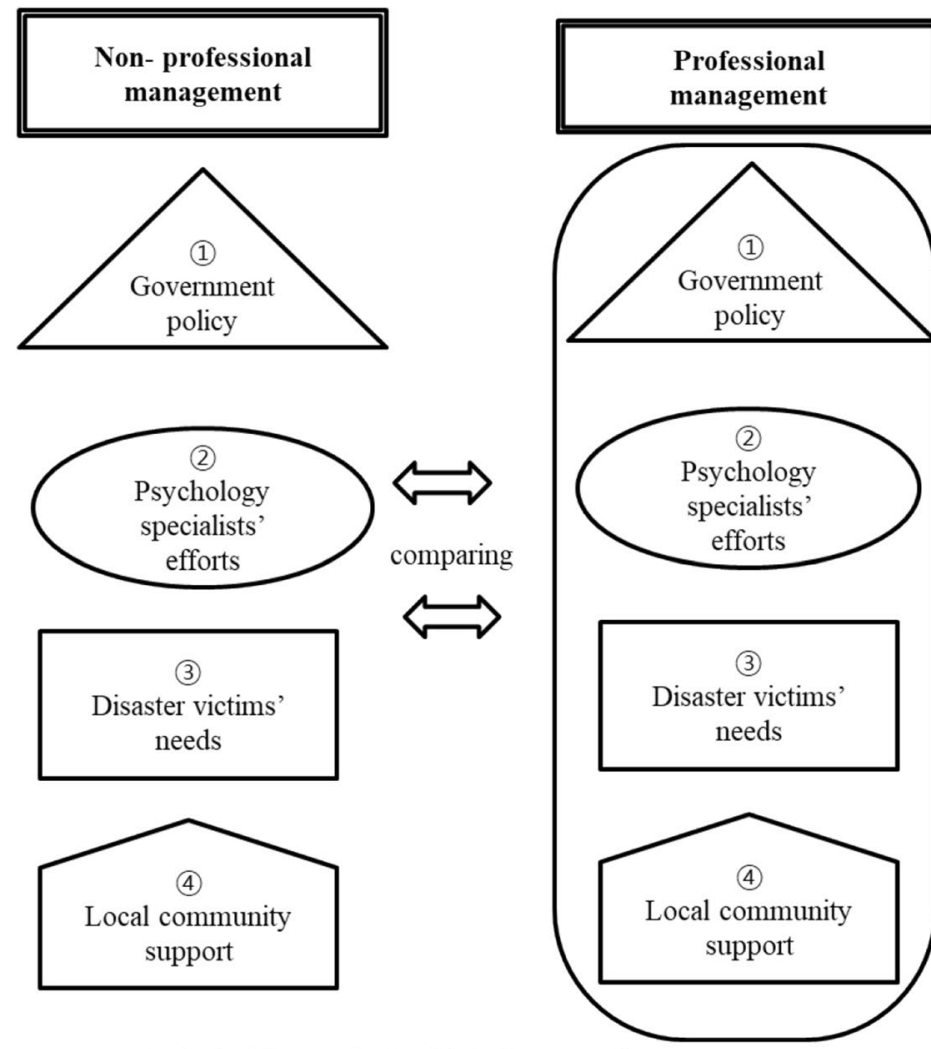

Psychological impact in the field of Korean disaster management
Methodology: qualitative content analysis

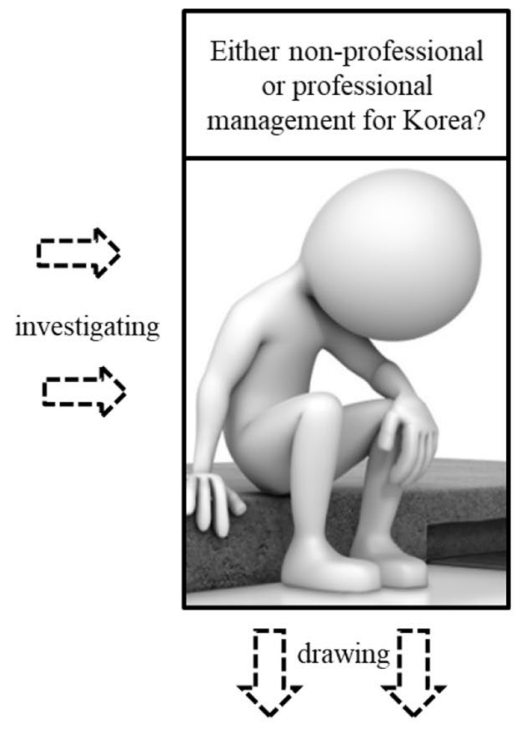

Lessons for neighboring nations (?)

Fig. 2 Research design

guidelines and allocated a budget for psychological impact management, the central government has yet to fully address the issue. As shown in Table 1, the policy guidelines are not very practical, and the budget allocation is small. In addition, 17 local governments have set up their own psychological support centers to manage the psychological impact of disasters within their local areas.

\section{Psychology Specialists' Efforts}

Psychology specialists in Korea include medical doctors, psychologists, psychiatrists, nurses, social workers, and counselors. After the sinking of the MV Sewol, the role of psychology specialists was seriously introduced to the public through mass media (Rush and Bloom 2014). However, these specialists have not successfully fulfilled their mission

Table 1 Examples of numerical data on nonprofessional management

\begin{tabular}{|c|c|}
\hline Units & Specific examples \\
\hline (1) Government policy & $\begin{array}{l}\text { - The national budget for trauma psychology was less than 1,600,000 USD in } 2018 \text { (total population = } 50 \text { mil- } \\
\text { lion) }\end{array}$ \\
\hline (2) Psychology specialists' efforts & $\begin{array}{l}\text { - Just after the oil leakage around Taean-gun in 2007, three residents without psychology specialists' support } \\
\text { committed suicide (similar cases continue to happen) } \\
\text { - After the outbreak of MERS in } 2015 \text {, only the health center in Guri city tried to provide psychological sup- } \\
\text { port for its residents }\end{array}$ \\
\hline (3) Disaster victims' needs & - About $10.4 \%$ of residents in Pohang city were provided with psychological support after earthquakes in 2017 \\
\hline (4) Local community support & $\begin{array}{l}\text { - In } 2019 \text {, more than } 58 \% \text { of local residents in Gyeonggi-do province did not know where to seek psychologi- } \\
\text { cal help } \\
\text { - Out of } 1.1 \text { million residents in Ulsan city, } 145 \text { local residents in } 2016 \text { and } 1036 \text { local residents in } 2017 \text { offi- } \\
\text { cially sought psychological support }\end{array}$ \\
\hline
\end{tabular}

Sources: (MOIS 2020) 
in recent emergencies. For instance, during the outbreak of avian influenza and foot and mouth disease in 2016 and 2017, respectively, only the amount of economic damage was considered.

\section{Disaster Victims' Needs}

The majority of Korean disaster victims are not fully aware of the concept of individual or group psychological impact. During a disaster, they generally focus on the human loss and economic damage without equal concern for coping with the psychological impact. At best, many disaster victims have just begun to recognize the psychological impact they are experiencing and have yet to determine how to deal with their situation.

\section{Local Community Support}

Local community support includes not only one-way but also two-way social support (that is, giving and taking) by relying on emotion, information, appraisal, and instruments between/among residents or people (Rung et al. 2017). In this context, local communities in Korea, such as neighborhoods, have played various roles in dealing with the psychological impact of disasters throughout history. For example, many community-based organizations, such as the Young Men's Group and the Married Women's Group, deal with such impact at the regional level.

\section{Professional Management of Psychological Impact}

\section{Government Policy}

The central government needs to provide professional policy guidelines on dealing with psychological impact in the context of disaster management. The current policy guidelines do not ask obligatory requirement on mitigating psychological impact but only a temporary one for the field, such as the COVID-19 outbreak in 2020. Local governments are considered as the first line of defense against disasters (Col 2007). Thus, it is necessary for the 17 local governments in Korea to fully implement their respective psychological support centers, in particular by hiring experts, applying their own strategies, and implementing long-term efforts.

\section{Psychology Specialists' Efforts}

Some psychology specialists have applied recent technologies to address the psychological impact of disasters in Korea. For instance, a group of psychology specialists used a computer system for the structured mandala (a ritual sign in Hinduism and Buddhism, which represents a circle or the universe) to identify individuals psychologically impacted by a disaster (Kim et al. 2014). This approach in psychological therapy has been standardized and is now being applied by other psychology specialists in some regions.

\section{Disaster Victims' Needs}

Disaster victims need to be aware of the issue of psychological impact within the context of disaster awareness. Without a partial or full understanding of the seriousness of the psychological impact of a disaster, the victims would not work toward overcoming their situation (Australian Red Cross 2012). That is, disaster victims need to prepare not only physically but also psychologically for various disasters, while experiencing the high extent of suffering.

\section{Local Community Support}

Although local communities have actively participated in coping with the psychological impact of disasters, all stakeholders need to undergo systematic psychological training (Woo et al. 2015). Several training programs on crisis intervention techniques are available for individuals, including informal defusing within $24 \mathrm{~h}$ after a disaster, critical incident stress debriefing, stress reduction techniques, and expressive techniques, particularly for children.

\section{Implications to Neighboring Nations}

The above comparison of the two management styles indicates that Korea should shift from its present nonprofessional approach to the professional style of disaster management. In the Korean setting, such professional management would be much more effective in dealing with the psychological impact of disasters. In particular, when thinking that the importance of each individual or related psychology has been raised in the twenty-first century regardless of national boundary, Korea has to explore new management style in the field.

The occurrence of a disaster may be considered as a window of opportunity because, paradoxically, it may potentially lead to reducing various risks in the region in the future (Cretney 2019). The present study holds that the occurrence of disasters in Korea can lead to the implementation of major changes or related improvements in many other nations by giving new insights on the psychological impact of disasters or providing the momentum to tackle this concern. Korea and its neighboring nations thus need to establish a collaborative relationship to efficiently address the issue. 
Besides, neighboring nations need to rationally approach the issue of the psychological impact of disasters. Any player in the field of disaster management who applies an emotional approach to this issue may end up not helping disaster victims overcome their situation (Mason et al. 2010). An emotional reaction will not solve any psychological problem. Thus, neighboring nations must approach the issue in a rational way.

The psychological impact of a disaster affects individuals on a personal level. Nevertheless, it is important for the whole nation to recognize the issue (Palinkas 2012). In Korea, only the local communities actively carry out their related roles and responsibilities. When a nation fully understands the psychological impact and then establishes the necessary steps to mitigate it, the extent of such impact in many individuals is decreased. Without a national effort, many individuals would not be able to dramatically reduce the psychological impact despite their own trials.

Similarly, each nation needs to approach the issue of psychological impact aggressively (World Bank 2002). Because the issue is easily disregarded during the short period of disaster response, its symptoms are rapidly aggravated. Hence, nations must consciously and aggressively approach the issue of psychological impact from its very onset.

The disaster management cycle includes four phases: (i) disaster prevention (e.g., monitoring of psychological change) and mitigation (e.g., psychology seminars), (ii) disaster preparedness (e.g., psychology training), (iii) disaster response (e.g., psychological therapy), and (iv) disaster recovery (e.g., psychological compensation). Some countries, including Korea, have not given equal attention to all four phases in terms of psychological impact. Instead, the focus has been on one or two phases only, particularly disaster response and recovery.

Neighboring nations have to equally address each phase of the disaster management cycle to efficiently tackle and minimize the psychological impact. These four phases exist throughout the whole duration of the disaster or the entire process of disaster management. Neglecting even just one of the four phases can quickly aggravate the extent of psychological impact.

In particular, neighboring nations must plan on how to deal with the issue of psychological impact through a series of emergency operation plans (EOPs), which can be regarded as a subphase of disaster preparedness (Elliott et al. 2017). In general, developed nations have included the issue of psychological impact in their EOPs more consistently compared with developing nations. By referring to it, all neighboring nations require the establishment of comprehensive EOPs in terms of psychological impact.

The psychological impact of disasters has clearly influenced the region over the years. The Great Eastern Japan earthquake in 2011 has left women and senior citizens in the area agonizing even to this day. The outbreak of COVID19 as a pandemic has psychologically impacted almost all nations in the world in unprecedented ways (WHO 2020). Thus, long-term measures to address the psychological impact of disasters must be implemented in the context of national disaster management.

\section{Conclusion}

The present study aimed to evaluate how Korea should enhance its management of the psychological impact of disasters and to draw lessons for neighboring nations regarding this issue. Two opposite management styles were presented: nonprofessional and professional. Several measures applicable to other nations were discussed, such as national efforts, aggressive management considering multiple factors, equal management of all four phrases of the disaster management cycle, and long-term planning. Therefore, the research objective was successfully achieved.

The key finding of this work is that Korea needs to transform its management style from nonprofessional to professional toward reducing the extent of the psychological impact of disasters. Toward this end, all four stakeholders, namely, governments, psychology specialists, disaster victims, and local communities, should carry out their assigned roles and responsibilities, as well as address the individual mentalities and nutritional needs within their cultural context. Neighboring countries must implement a comprehensive approach to the psychological impact of disasters without neglecting the roles and responsibilities of these various stakeholders.

Despite its limitations, this research has value in that, compared with previous studies, it applies a more comprehensive or rigorous approach to the issue of psychological impact in Korea from the perspective of disaster management. Whereas some researchers have studied disaster psychology strictly within the field of trauma psychology, there is a lack of similar research from the perspective of disaster management. Not only the psychological perspective but also a whole community perspective should have been utilized more.

The field of disaster management in Korea needs to expand its research on the psychological impact of disasters. Although the present work has provided an analytical framework for the study of such impact, future investigations may further explore all suggested variables, limitations, and alternatives. In addition, regarding the transition from a nonprofessional to a professional management style, researchers in neighboring nations should evaluate the issue of psychological impact in their respective locations by applying more restrictive criteria of disaster management. 


\section{Compliance with Ethical Standards}

Conflict of interest The author declares that he has no conflict of interest.

\section{References}

Ansan Mental Health Trauma Center. (2017). Support for emergency psychology (in Korean). Ansan Mental Health Trauma Center. Retrieved January 2, 2020, from https://www.ansantrauma.net/

Australian Red Cross. (2012). Psychological preparedness for disasters. North Melbourne: Australian Red Cross.

Belzen, J. A. (2019). What, why and how? Meta-reflections on cultural psychological approaches to the scientific study of phenomena called religious. Integrative Psychological and Behavioral Science, 53(1), 158-187. https://doi.org/10.1007/s12124-018-9427-9.

Chae, E.-H., Kim, T.-W., Rhee, S.-J., \& Henderson, T. D. (2005). The impact of flooding on the mental health of affected people in South Korea. Community Mental Health Journal, 41(6), 633-645. https://doi.org/10.1007/s10597-005-8845-6.

Choi, J.-Y., \& Oh, K.-J. (2014). Cumulative childhood trauma and psychological maladjustment of sexually abused children in Korea: Mediating effects of emotion regulation. Child Abuse \& Neglect, 38(2), 296-303. https://doi.org/10.1016/j.chiabu.2013.09.009.

Col, J.-M. (2007). Managing disasters: The role of local governments. Public Administration Review. https://doi.org/10.111 1/j.1540-6210.2007.00820.x.

Cox, R. S., Scannell, L., Heykoop, C., Tobin-Gurley, J., \& Peek, L. (2017). Understanding youth disaster recovery: The vital role of people, places, and activities. International Journal of Disaster Risk Reduction, 22, 249-256. https://doi.org/10.1016/j.ijdrr .2017.03.011.

Cretney, R. (2019). "An opportunity to hope and dream": Disaster politics and the emergency of possibility through communityled recovery. Antipode, 51(2), 497-516. https://doi.org/10.1111/ anti.12431.

Elliott, L., Benoit, E., Matusow, H., \& Rosenblum, A. (2017). Disaster preparedness among opioid treatment programs: Policy recommendations from state opioid treatment authorities. International Journal of Disaster Risk Reduction, 23, 152-159. https://doi. org/10.1016/j.ijdrr.2017.05.001.

Gelo, O., Braakmann, D., \& Benetka, G. (2008). Quantitative and qualitative research: Beyond the debate. Integrative Psychological and Behavioral Science, 42(3), 266-290. https://doi.org/10.1007/ s12124-008-9078-3.

Gray, M. J., Shira, M., \& Litz, B. T. (2004). Acute psychological impact of disaster and large-scale trauma: Limitations of traditional interventions and future practice recommendations. Prehospital and Disaster Medicine, 19(1), 64-72. https://doi.org/10.1017/S1049 $023 X 00001497$.

Hall, S. (2017). How to Do Content Analysis. Classroom Driven by Leaf Group. Retrieved December 22, 2019, from https://class room.synonym.com/content-analysis-2670.html

Han, K.-M., Kim, K.-H., Lee, M.-K., Lee, S.-M., Ko, Y.-H., \& Paik, J.-W. (2017). Increase in the prescription rate of antidepressants after the Sewol Ferry disaster in Ansan, South Korea. Journal of Affective Disorder, 219, 31-36. https://doi.org/10.1016/j. jad.2017.05.026.

Hu, X., Yang, Y., Liu, L., Liu, X., \& Tong, Y. (2010). Early psychological intervention following a natural disaster: A study with a victim buried under rubble for 124 hours. Social Behavior and Personality, 38(1), 71-74. https://doi.org/10.2224/sbp.2010.38.1.71.
Jogia, J., Kulatunga, U., Yates, G. P., \& Wedawatta, G. (2014). Culture and the psychological impacts of natural disasters: Implications for disaster management and disaster mental health. The Built \& Human Environment Review, 7(1), 1-10.

Kaplan, B. J., Rucklidge, J. J., Romijn, A. R., \& Dolph, M. (2015). A randomised trial of nutrient supplements to minimize psychological stress after a natural disaster. Psychiatry Research, 228(3), 373-379. https://doi.org/10.1016/j.psychres.2015.05.080.

Kim, S.-I., Ghil, J.-H., Choi, E.-Y., Kwon, O.-S., \& Kong, M. (2014). A computer system using a structured mandala to differentiate and identify psychological disorders. The Arts in Psychotherapy, 41(2), 181-186. https://doi.org/10.1016/j.aip.2014.02.003.

Lebowiz, A. J. (2017). Relational satisfaction from providing and receiving support is associated with reduced post-disaster depression: Data from within one year of the 2011 Japan triple disaster. Community Mental Health Journal, 53, 202-214. https://doi. org/10.1007/s10597-016-9995-4.

Lindell, M. K., \& Prater, C. S. (2003). Assessing community impacts of natural disasters. Natural Hazards Review, 4(4), 176-185. https ://doi.org/10.1061/(ASCE)1527-6988(2003)4:4(176).

Mason, V., Andrews, H., \& Upton, D. (2010). The psychological impact of exposure to floods. Psychology, Health \& Medicine, 16(1), 61-73. https://doi.org/10.1080/13548500903483478.

McGonagle, L. C. (1964). Psychological aspects of disaster. American Journal of Public Health, 54(4), 638-643. https://doi.org/10.2105/ AJPH.54.4.638.

Ministry of the Interior and Safety (MOIS). (2020). Official Website of MOIS (in Korean). Ministry of the interior and safety. Retrieved July 1, 2020, from https://www.mois.go.kr/frt/a01/frtMain.do

Nomura, S., Parsons, A. J. Q., Hirabayashi, M., Kinoshita, R., Liao, Y., \& Hodgson, S. (2016). Social determinants of mid-to longterm disaster impacts on health: A systematic review. International Journal of Disaster Risk Reduction, 16, 53-67. https://doi. org/10.1016/j.ijdrr.2016.01.013.

Othman, S.-H., \& Beydoun, G. (2013). Model-driven disaster management. Information \& Management, 50(5), 218-228. https://doi. org/10.1016/j.im.2013.04.002.

Palinkas, L. A. (2012). A conceptual framework for understanding the mental health impacts of oil spills: Lessons from the Exxon Valdez oil spill. Psychiatry, 75(3), 203-222. https://doi.org/10.1521/ psyc.2012.75.3.203.

Rung, A. L., Gaston, S., Robinson, W. T., Trapido, E. J., \& Peters, E. S. (2017). Understanding the disaster-depression knot: The role of social ties after Deepwater Horizon. Social Science \& Medicine, 177, 19-26. https://doi.org/10.1016/j.socscimed.2017.01.041.

Rush, J. \& Bloom, D. (2014). Ferry victims' last, horrifying moments: Corpses pulled from Korea wreck reveal children broke the fingers trying to escape sinking ship. Retrieved May 1, 2020, from https ://www.dailymail.co.uk/news/article-2611159/Haunting-image s-memorial-victims-South-Korea-ferry-disaster-photographs-leftloved-ones-identified.html

Smith, L. E., Bernal, D. R., Schwartz, B. S., Whitt, C. L., Christman, S. T., Donnelly, S., et al. (2014). Coping with vicarious trauma in the aftermath of a natural disaster. Journal of Multicultural Counseling and Development, 42(1), 1-12. https://doi.org/10.10 02/j.2161-1912.2014.00040.x.

Stopper, S. (2017). Non-Professional vs. Professional Jobs. eHow. Retrieved June 4, 2020, from https://www.ehow.com/info_81406 75_nonprofessional-vs-professional-jobs.html

Swann, C., Crust, L., \& Allen-Collinson, J. (2016). Surviving the 2015 Mount Everest disaster: A phenomenological exploration into lived experience and the role of mental toughness. Psychology of Sport and Exercise, 27, 157-167. https://doi.org/10.1016/j.psych sport.2016.08.012.

Tachibana, A., Kitamura, H., Shindo, M., Honma, H., \& Someya, T. (2014). Psychological distress in an earthquake-devastated 
area with pre-existing high rate of suicide. Psychiatry Research, 219(2), 336-340. https://doi.org/10.1016/j.psychres.2014.01.028.

Tanaka, E., Tsutsumi, A., Kawakami, N., \& Kameoka, S. (2016). Longterm psychological consequences among adolescent survivors of the Wenchuan earthquake in China: A cross-sectional survey six years after the disaster. Journal of Affective Disorders, 204, 255-261. https://doi.org/10.1016/j.jad.2016.08.001.

West, N. T., \& Albrecht, K. (2007). Building emotional competence: A strategy for disaster preparation and recovery. Exchange: The Early Childhood Leaders' Magazine Since 1978, 175, 20-25.

Whittle, R., Walker, M., Medd, W., \& Mort, M. (2012). Flood of emotions: Emotional work and long-term disaster recovery. Emotion Space and Society, 5(1), 60-69. https://doi.org/10.1016/j.emosp a.2011.08.002.

Woo, H.-K., Cho, Y.-T., Shim, E.-Y., Lee, K.-H., \& Song, G.-Y. (2015). Public trauma after the Sewol ferry disaster: The role of social media in understanding the public mood. International Journal of
Environmental Research and Public Health, 12(9), 10974-10983. https://doi.org/10.3390/ijerph120910974.

World Bank. (2002). Natural Hazard Risk Management in the Caribbean: Revisiting the Challenge. World Bank. Retrieved June 21, 2020, from https://siteresources.worldbank.org/INTDISMGMT/ Resources/cgced_final.pdf

World Health Organization (WHO). (2020). Mental health and psychosocial considerations during the COVID-19 outbreak. World Health Organization. Retrieved June 11, 2020, from https://www. who.int/docs/default-source/coronaviruse/mental-health-consi derations.pdf

Publisher's Note Springer Nature remains neutral with regard to jurisdictional claims in published maps and institutional affiliations. 